\title{
THREE-DIMENSIONAL STRUCTURE OF DENDRITIC SPINES IN THE RAT NEOSTRIATUM ${ }^{1}$
}

\author{
C. J. WILSON, ${ }^{*, 2}$ P. M. GROVES, $\neq$ S. T. KITAI,* AND J. C. LINDER \\ * Department of Anatomy, Michigan State University, East Lansing, Michigan 48824 and $\$$ Department of Psychiatry, \\ University of California, San Diego, La Jolla, California 92093
}

Received May 24, 1982; Revised September 29, 1982; Accepted September 30, 1982

\begin{abstract}
Dendritic spines of rat neostriatal neurons were examined by light microscopy and high voltage stereo electron microscopy (HVEM) following selective staining by intracellular microinjection of horseradish peroxidase. Conventionally prepared material also was used for quantitative analysis of dendritic spines from serial thin sections of neostriatum. Stereo electron microscopy of semithin sections from rat neostriatum fixed using a protocol designed to preserve cytoskeletal integrity was employed to examine the organization of the dendritic spine cytoplasm.

Light microscopic and HVEM examination of spiny dendrites and quantitative analysis of serial thin sections from normal material revealed no distinct spine types but rather continuous and independent variation of spine head diameter, stalk diameter, and stalk length. Likewise, there was no systematic relationship between any of these spine dimensions and dendritic diameter. Spine head membrane surface area was directly related to the area of the synaptic junctional membrane of the spine head. In semithin sections, the cytoplasm of the spine contained membranous saccules of spine apparatus and a delicate cytoskeletal network composed of microfilaments and a set of finer and more variable cytoskeletal filaments. It is proposed that this cytoskeletal network together with the spine apparatus is responsible for the maintenance and alteration of spine shape and in this way controls the effectiveness of axospinous synapses.
\end{abstract}

Dendritic spines arc a common form of postsynaptic specialization found on many types of neurons in widespread regions of the central nervous system. In certain brain structures, most noticeably in cerebral and cerebellar cortices, hippocampus, and neostriatum, axospinous synaptic contacts are highly concentrated on one or more particular populations of neurons and may represent the predominant type of interneuronal junction on those cells. Likewise, certain afferent fiber systems in these brain regions tend to contact dendritic spines preferentially, while others are excluded from making such

'A preliminary report of these experiments was presented at the 20th Annual Meeting of the American Society for Cell Biology, November 1980. This work was supported by National Institute of Neurological and Communicative Disorders and Stroke Grant NS 17294 (to C. J. W.), National Institute of Drug Abuse Grants DA 02854 and Research Scientist Development Award DA 00079 and a grant from the John D. and Catherine T. MacArthur Foundation (to P. M. G.), National Institute of Neurological and Communicative Disorders and Stroke Grant NS 14866 (to S. T. K.), and Grant RR-00592 to the high voltage electron microscopy facility at the University of Colorado. We thank Dr. E. Fifková for reading the manuscript and for helpful comments throughout the course of this work.

${ }^{2}$ To whom correspondence should be addressed. synapses. This rigid arrangement of synapses has suggested to many investigators that dendritic spines may play a functional role in the integration of afferent activity in those neuron types characterized by a high density of axospinous contacts (Chang, 1952; Diamond et al., 1970; Llinás and Hillman, 1969; Rall, 1974; Ramon y Cajal, 1911; Shepherd and Brayton, 1979). Supportive evidence for the functional importance of dendritic spines has come from descriptive studies of alterations in spine morphology upon changes in environmental conditions (Coss and Globus, 1978; Globus and Scheibel, 1967; Valverde, 1967), central nervous system pathology (Purpura, 1974), or experimentally induced alteration of axospinous synaptic transmission (Fifková and Van Harreveld, 1977; Van Harreveld and Fifková, 1975). The possibility that dendritic spine shape and size may directly determine the effectiveness of synaptic transmission at individual synapses has been proposed from theoretical consideration of the passive electrical properties expected to result from such geometrical arrangements of neuronal membrane (Diamond et al., 1970; Jack et al., 1975; Rall, 1974; Shepherd and Brayton, 1979). Observations of morphological changes accompanying experimentally produced long-term synaptic poteritiation in the hippocampus have demonstrated a temporal coincidence of spine shape 
alteration and changes in the strength of synaptic transmission (Fifková and Anderson, 1981; Fifková and Van Harreveld, 1977; Van Harreveld and Fifková, 1975). (For an alternative view, however, see Lee et al., 1980.)

Regardless of whether changes in dendritic spine size or shape do occur under normal circumstances, it is possible that rather small constant differences in spine geometry could influence the effectiveness of transmission at individual synapses on a neuron. Systematic differences in spine distribution and geometry have been reported for different regions of dendritic trees of some neurons in the hippocampus (Laatch and Cowan, 1966), cerebral cortex (Jones and Powell, 1969; Peters and Kaiserman-Abramof, 1970), and optic tectum of fish (Coss and Globus, 1978). In all of these cases, however, the dendritic trees of the spiny neurons cross through the axonal ficlds of different afferent systems. Thus differences in the geometry of spines placed on small distal dendrites and those on larger proximal portions of the dendritic tree might be related to position on the neuron, dendritic diameter, or the nature of the afferent innervation at that level.

A less complicated arrangement for the study of axospinous synapses is available in the mammalian neostriatum. This region is characterized by the preponderance of a single morphological cell type possessing a high density of dendritic spines. The three largest sources of extrinsic afferents to neostriatum, the cerebral cortex, intralaminar thalamic nuclei, and substantia nigra, form most of their contacts with the spines of this neuron (Chung et al., 1977; Groves, 1980; Hassler, et al., 1978; Hattori et al., 1973; Kemp and Powell, 1971c, d). At least the cortical and thalamic afferents appear to share a common distribution along the dendrites of the cells (Kemp and Powell, 1971d), which themselves have no preferred orientation (DiFiglia et al., 1976; Fox et al., 1971/1972; Kemp and Powell, 1971a; Preston et al., 1980; Ramon y Cajal, 1911; Wilson and Groves, 1980). The dendritic spines of the neurons apparently are identical in structure to those of the more elaborate spiny neurons found in the cerebral cortex (Kemp and Powell, 1971b; Pasik et al., 1976; Tarrant and Routtenberg, 1977).

In the experiments reported here, the distribution, shape, shape variations, synaptic arrangements, and cytoplasmic components of dendritic spines have been examined in material prepared from rat neostriatum using a variety of techniques. Variation of shape and size parameters which could affect synaptic transmission was analyzed quantitatively, and a possible cellular mechanism for the maintenance and alteration of spine shape is presented.

\section{Materials and Methods}

Intracellularly labeled neurons. Rat neostriatal neurons were stained intracellularly with horseradish peroxidase (HRP) in the course of intracellular recording of their spontaneous and evoked electrical activity (Chang et al., 1981; Wilson and Groves, 1980, 1981). Brains from these animals were fixed by intracardial perfusion with a mixture of aldehydes in $0.15 \mathrm{M}$ phosphate buffer $(\mathrm{pH}$ 7.4) and Vibratome sections through the neostriatum were collected and processed for demonstration of peroxidase activity. Sections containing injected neurons were postfixed in osimium tetroxide and section embedded in Spurr's resin for sequential light and electron microscopy as described elsewhere (Chang et al., 1981; Wilson and Groves, 1979).

Light microscopic analyses of dendritic spine distributions were performed on a Leitz Orthoplan microscope using a $\times 100$ oil immersion objective and a $\times 10$ measuring eyepiece. Slides and coverslips were removed from sections intended for high voltage electron microscopy. Areas containing intracellularly stained neurons were remounted on blocks of plastic and sectioned on an ultramicrotome at 3 to $5 \mu \mathrm{m}$ using a dry glass knife (Favard and Carasso, 1973). Sections containing spiny dendritic processes were mounted on 50 mesh folding copper grids and coated on both sides with carbon. They were examined unstained at $1000 \mathrm{kV}$ on the high voltage electron microscope at the University of Colorado at Boulder.

Serial sections. Serial thin sections were obtained from blocks of rat neostriatum fixed in $4 \%$ formaldehyde $1 \%$ glutaraldehyde in $0.15 \mathrm{~m}$ phosphate buffer ( $\mathrm{pH} 7.4$ ), postfixed in $1 \%$ osmium tetroxide, stained en bloc in $0.5 \%$ aqueous uranyl acetate, and embedded in Epon/Araldite. Ribbons of from 30 to 100 consecutive sections were mounted on Formvar-coated slotted grids and stained with lead citrate (Venable and Coggeshall, 1965). Dendritic spines to be used in the quantitative analysis were analyzed from photographs printed with a final magnification of $\times 25,000$ and were required to be completely contained within a series of photographs from consecutive sections. Profile cross-sectional areas, synaptic appositional lengths, and perimeters were measured using a digitizing tablet connected to a PDP-11 computer. Volumes and surface areas were calculated using an average section thickness for the series determined by using section interference colors.

Semithin sections. For analysis of the organization of the dendritic spine cytoplasm, rats were heavily anesthetized with sodium pentobarbital and perfused intracardially with warm $\left(37^{\circ} \mathrm{C}\right) \mathrm{Krebs}$-Ringer solution containing $135 \mathrm{~mm} \mathrm{NaCl}, 5 \mathrm{~mm} \mathrm{KCl}, 1 \mathrm{~mm} \mathrm{MgCl}, 1.25 \mathrm{~mm}$ $\mathrm{Na}_{2} \mathrm{HPO}_{4}, 15 \mathrm{~mm} \mathrm{NaHCO}, 11 \mathrm{~mm}$ glucose, 2 mM EDTA, and $0.1 \%$ hydrogen peroxide and $0.33 \mathrm{gm} / \mathrm{liter}$ of GTP added just before use. The solution was brought to $\mathrm{pH}$ 7.2 just before use by bubbling with $95 \%$ oxygen $/ 5 \%$ carbon dioxide and was perfused for a period of from 1 to $5 \mathrm{~min}$ prior to fixation. This solution was followed by perfusion with $2 \%$ glutaraldehyde/ $2 \%$ formaldehyde in the same solution for 15 to $20 \mathrm{~min}$, after which the brain was removed and left in fixative overnight at $4^{\circ} \mathrm{C}$. Blocks from neostriatum were removed and postfixed for $1 \mathrm{hr}$ in a solution of $1 \%$ osmium tetroxide $/ 1.5 \%$ potassium ferricyanide in $0.15 \mathrm{M}$ cacodylate buffer ( $\mathrm{pH}$ 7.2) (Langford and Coggeshall, 1979). Blocks then were stained en bloc with $0.5 \%$ aqueous uranyl acetate for 12 to $16 \mathrm{hr}$, dehydrated in ethanol and propylene oxide, and embedded in Epon/Araldite. Sections ranging from 100 to $250 \mathrm{~nm}$ in thickness were mounted on uncoated 300 mesh grids, stained in aqueous uranyl acetate and lead citrate, and examined at 100 or $1000 \mathrm{kV}$. 


\section{Results}

Distribution of dendritic spines. The characteristic features of spiny neostriatal neurons as observed after intracellular HRP injection have been described elsewhere (Chang et al., 1981; Preston et al., 1980; Wilson and Groves, 1980). The most useful single characteristic for identification of these neurons is the very heavy investment of dendritic spines on the distal dendrites and their absence on the most proximal dendrites and somata. An example showing this characteristic distribution of dendritic spines is shown in Figure 1a. The photomontage in that figure shows a smooth dendrite arising from the soma of an injected neuron and bifurcating within the first few micrometers of its course. One of the resulting branches, which is maintained in focus in the photomontage, is unbranched over the remainder of its length. As indicated in that example, the dendriles of spiny neurons generally were between 0.5 and $1.0 \mu \mathrm{m}$ in diameter, tapered very gradually with length, and underwent their major diameter changes at branch points and near the end of the dendrites. In contrast to dendritic diameter, spine density was very sensitive to distance from the soma, increasing rapidly over the initial spiny portion of the dendrite to reach a peak value, after which there was a steady decline. This pattern of dendritic spine density is the same qualitatively as that reported previously for spiny neostriatal neurons in the cat (Kemp and Powell, 1971d) and monkey (Pasik et al, 1976). Quantitative measurement of dendritic spine densities at various parts of the dendritic field was performed on five spiny neurons for which at least four dendrites were completely contained in a single $50-\mu \mathrm{m}$ section containing the soma. Although possibly introducing some bias in favor of short dendrites, this restriction made it possible to measure spine densities with only occasional small corrections for dendritic foreshortening due to variation in depth within the plane of the section. Spine heads were counted for $10-\mu \mathrm{m}$ segments beginning from the soma and continuing to the end of the dendrite. At least four dendrites were studied for each neuron included in the sample to allow assessment of variation in spine density along individual dendrites, across dendrites on the same neuron, and among neurons. Because of the small diameter of the dendrites of striatal neurons and because the HRP-labeled dendrites used for these measurements were not entirely opaque, spines arising from all angles appeared to be visible, suggesting that correction for the proportion of spines obscured by the dendrite might not be required. The extent to which spine densities are underestimated due to the silhouette of the dendrite in tissue sections depends upon dendritic diameter and on the length and diameter of the spines (Feldman and Peters, 1979). Since these all may vary systematically with distance from the soma, the extent to which such errors of cstimation occur is of importance for quantitative studies. Examples showing the appearance of the dendrites and spines as observed through focus are shown in Figure $1, b$ to $d$. Figure $1 b$ shows micrographs taken at three focal planes through a dendritic termination. Several dendritic spines of various morphology arise from the final, sharply tapered portion of the dendrite. The dendritic segment shown in Figure $1 c$ was located at a distance of approximately 70 $\mu \mathrm{m}$ from its cell body of origin and illustrates the appearance of a dendrite at its region of maximal spine density. Although the dendrite at this level is of somewhat larger diameter than the one shown in Figure $1 b$ (about $1 \mu \mathrm{m}$ ), the micrographs taken at focal levels on both sides of the dendritic shaft reveal dendritic spines arising at all angles around the dendrite. In Figure 1d, the appearance of a dendrite near its origin is shown. In this initial spine-sparse region of the dendrite, dendritic diameter and opacity are both high and the accuracy of spine density estimates is much more doubtful. Spine densities approach zero in this region, however, making such an error relatively insignificant for the overall distribution of dendritic spines (Fig. 2).

The results of spine density measurements for five neurons are shown in Figure 2. In all cells, the same basic pattern of spine distribution was obtained. Spine densities were extremely low ( 0 to $4 / 10 \mu \mathrm{m}$ ) over the first 20 $\mu \mathrm{m}$ of dendritic length for all cells, with an overall mean of $0.5 / 10 \mu \mathrm{m}$ of dendrite. Dramatic increases always were observed in the region of from 20 to $60 \mu \mathrm{m}$ from the soma, with peak spine density occurring at a distance of from 50 to $100 \mu \mathrm{m}$. The overall shape of the spine distribution was simple and unimodal, and spine densities always decreased with distance from the soma in the distal portions of the dendritic field.

Variation among dendrites on individual neurons and variation among neurons can also be seen in Figure 2. Variation among dendrites on single neurons was surprisingly small. In contrast, variation in overall spine density was quite marked among neurons, with peak spine densities varying from 22 to $40 / 10 \mu \mathrm{m}$ of dendritic length (Fig. 2f).

Spine shape and shape variation. Light microscopic examination of dendritic spines revealed no systematic variation of spine shape or size along the dendrites of the neurons. While there were occasional spines observed on the usually spine-free portions of the neurons (somata, axon initial segments, and proximal dendrites) and these were smaller and more sessile than those found in spiny regions of the dendrites, spines of all sizes and shapes were found at all positions along the heavily spine-laden portion of the dendrites (e.g., Fig. 1a). Most spines appeared to be simple and pedunculated in shape with clearly defined rounded heads and relatively long, thin stalks. The sizes of both stalk and head regions varied noticeably from spine to spine, but the resolution of the light microscopic preparation was insufficient for a quantitative analysis of spine shape.

To obtain the necessary resolution' in HRP-injected neurons, some labeled spiny dendrites were examined in 3 - to 5 - $\mu \mathrm{m}$-thick sections prepared for high voltage electron microscopy. Because the material used for this purpose had been postfixed in osmium tetroxide, the HRP reaction product was heavily impregnated with osmium black and the HRP-labeled cell processes were strongly electron dense. Unlabeled tissue surrounding the selectively stained elements showed a moderate density due to osmium fixation, but only the most heavily osmiophilic tissue landmarks could be recognized (e.g., myelinated fibers) due to the section thickness and the absence of other electron-dense stains. Examples of spiny 

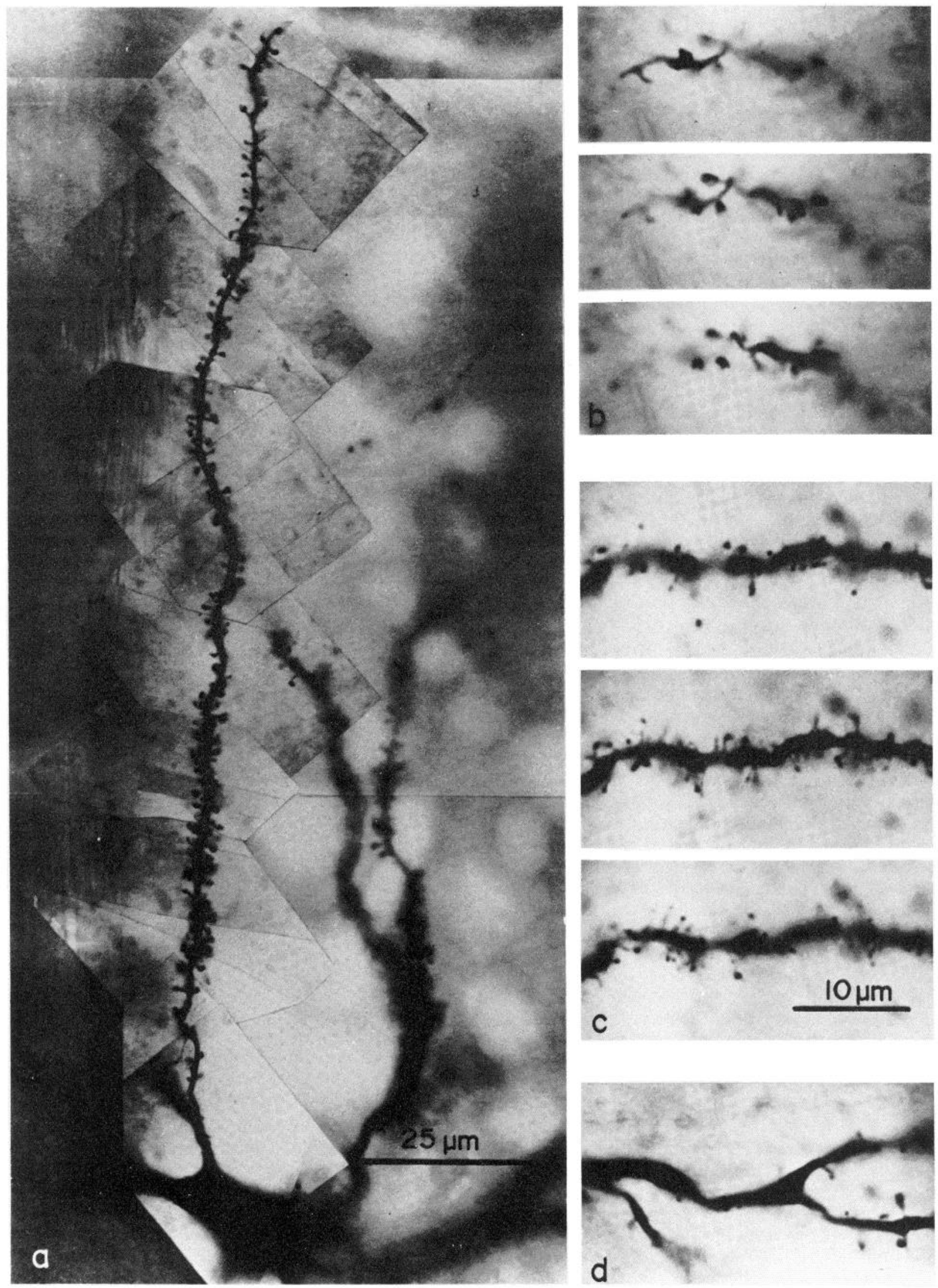

C

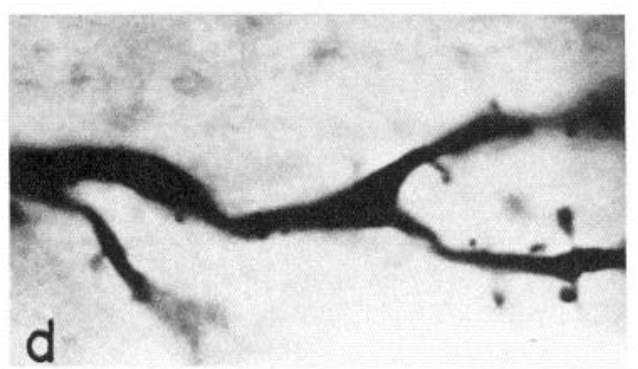

Figure 1. Light microscopic appearance of dendritic spines on intracellularly stained neostriatal spiny neurons. $a$, Photomontage showing the entire length of one dendrite from an HRP-injected neuron. The characteristic distribution of dendritic spines and the variety of spine shapes and sizes are apparent. $b$, Photomicrographs taken at three focal planes through the tip of a dendrite, showing the dramatic tapering of the dendrite and the appearance of dendritic spines at high magnification $(\times 2000) . c$, A similar series from the midregion of a dendrite at the region of highest spine density. $d$, The proximal region of a spiny dendrite at the level of the first spines. 
dendrites prepared for stereo HVEM analysis are shown in Figures 3 and 4. Stereo HVEM of intracellularly labeled neurons provided an accurate and convenient means for the assessment of linear spine dimensions (i.e., head diameter, neck diameter, and neck length). Analysis of these dimensions of spine geometry required the use of stereoscopic measurements but was relatively unaffected by the irregular shape of the spines. The semitransparent appearance of labeled dendrites allowed visualization of spines arising from all angles to the direction of view (Figs. 3 and 4).

With the improved resolution of the three-dimensional structure of the spines, an unexpected variety of spine morphology was encountered. Branched spines and tortuously curved spines were seen to be relatively common (Fig. 3). Spine necks with diameters ranging from slightly less than $0.1 \mu \mathrm{m}$ to about $0.5 \mu \mathrm{m}$ and lengths ranging from 0.35 to $3.80 \mu \mathrm{m}$ were seen. Spine heads ranged in diameter from 0.11 to $0.95 \mu \mathrm{m}$. Although direct measurement of distance from the soma was not made for these dendritic segments, no systematic variation in spine morphology could be seen to be associated with indirect
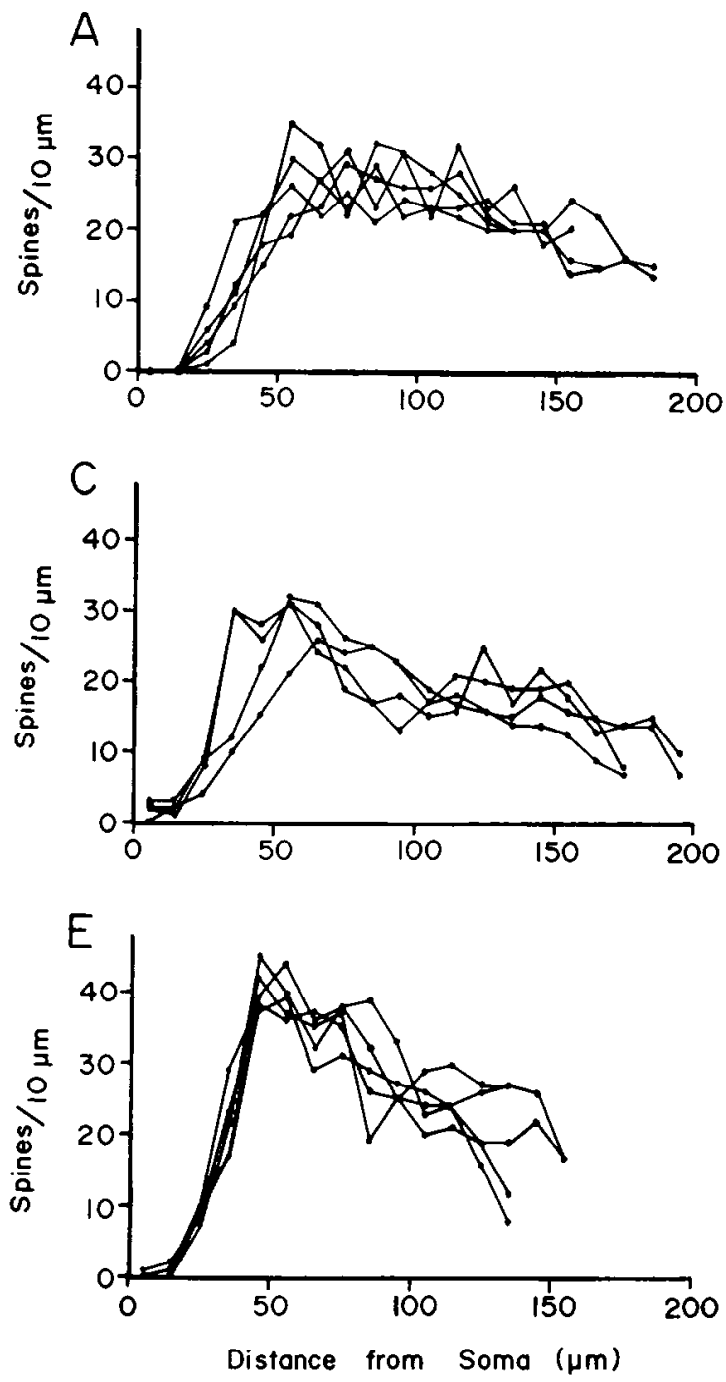

indicators of distance from the soma, such as dendritic diameter or spine density. Maximal spine densities could be measured with great accuracy from these high resolution three-dimensional images and ranged as high as $46 / 10 \mu \mathrm{m}$, in agreement with the results obtained from the light microscopic analysis.

Spine types. Three distinct spine types have been described from light and electron microscopic observations of spines in cerebral cortex (Peters and KaisermanAbramof, 1970) and monkey neostriatum (Pasik et al., 1976). These types, thin or pedunculated, sessile, and mushroom-shaped, are distinguished primarily on the basis of the length and diameter of the stalk in comparison with the size of the head. Further classification may be made on the basis of head shape (Jones and Powell, 1969). While clear examples of each of these spine types could easily be found in stereo HVEM or thin section images of striatal spiny dendrites (illustrated in Fig. 5), there was a variety of spines of intermediate morphology. It could not be determined from these qualitative observations whether spines of neostriatal neurons are best considered as belonging to one of three discrete catego-
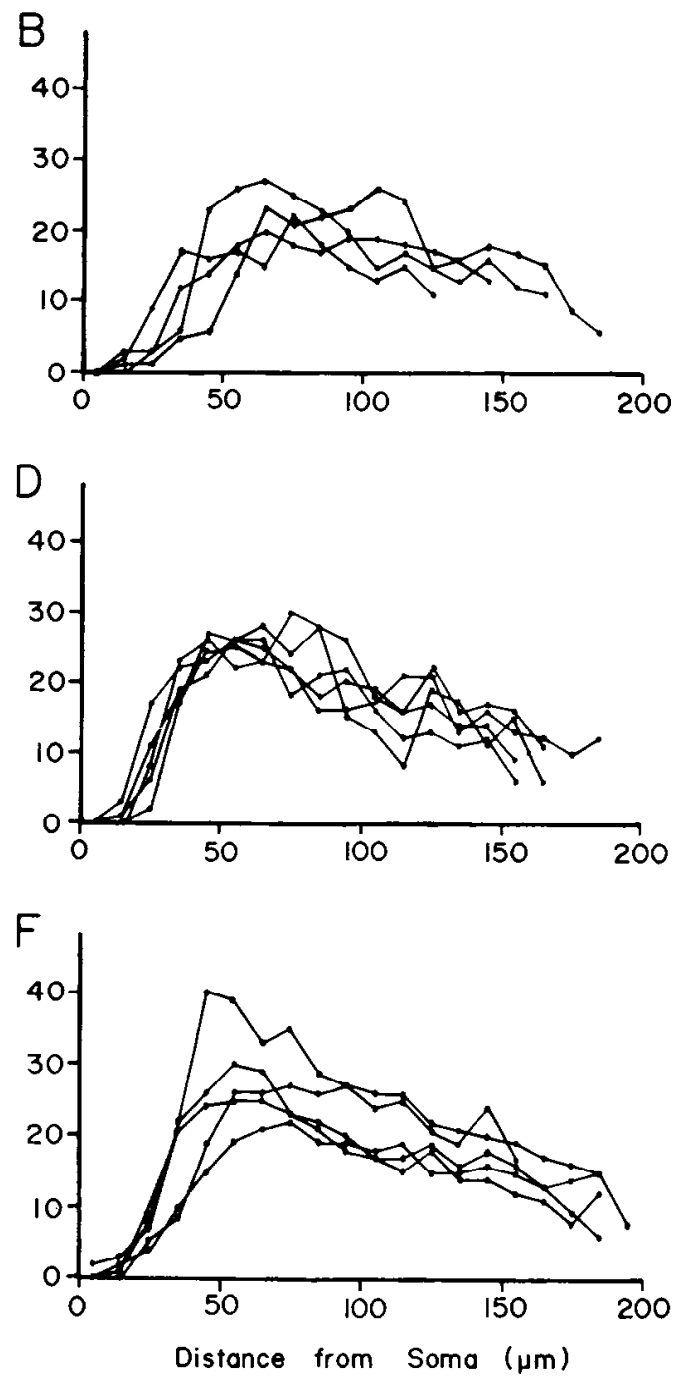

Figure 2. Spine density measurements for five intracellularly stained spiny neurons ( $A$ through $E$ ). Individual curves represent separate dendrites sampled from each neuron to assess variation within neurons. The mean distributions for each of the neurons shown are superimposed in $F$. 

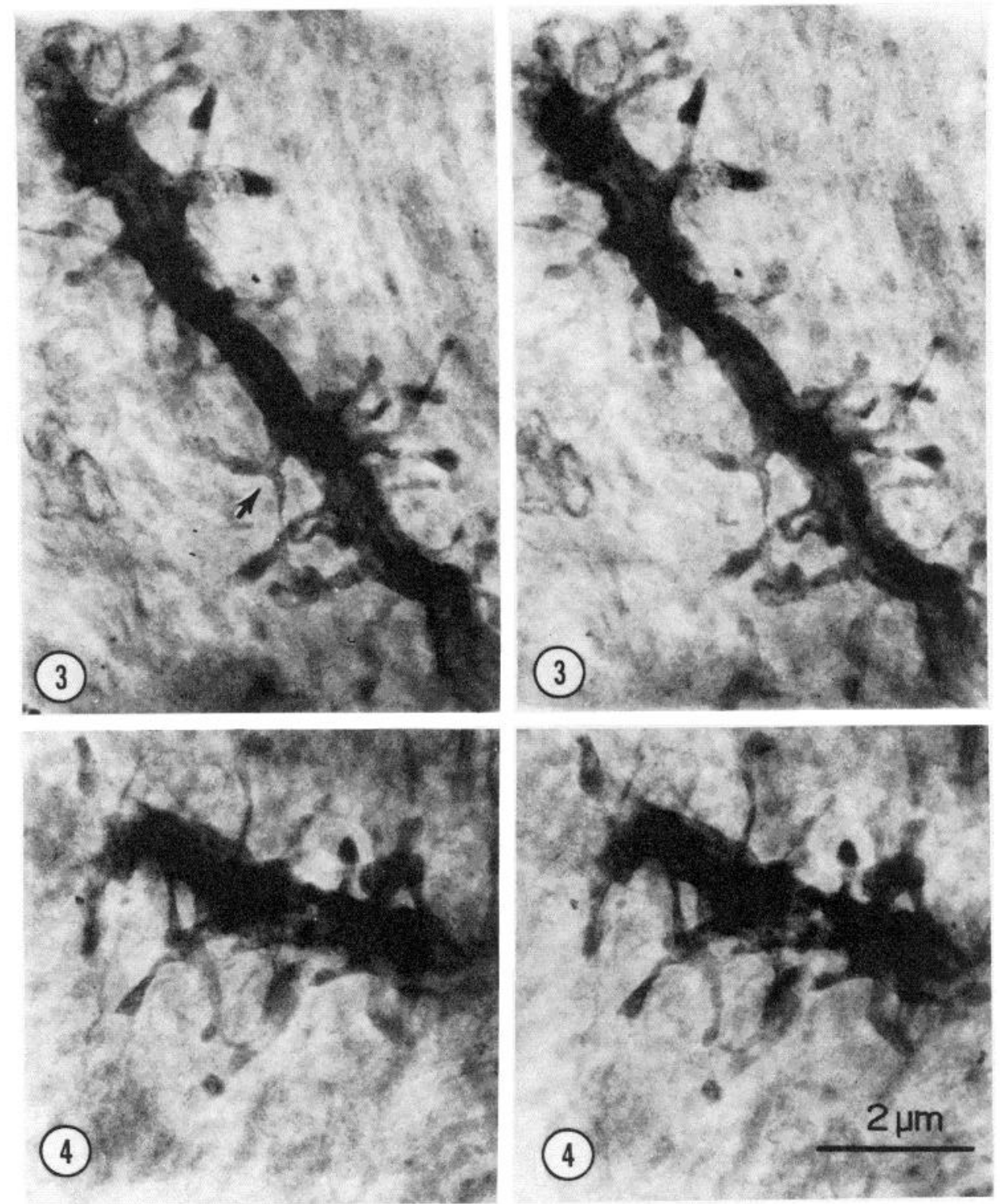

Figures 3 and 4. High voltage stereo electron micrographs of spiny dendrites from intracellularly stained neurons. The increased resolution of surface morphology obtained in these images allows identification of branched spines (arrow) and reveals a wide variety of spine shapes. Magnification $\times 10,000$; section thickness, $5 \mu \mathrm{m}$; tilt, $\pm 4^{\circ}$. Intracellular staining is somewhat nonuniform, due to the exclusion of HRP from the interior of membrane-bound organelles. Especially visible are mitochondria, which appear as elongated, lightly stained areas in the dendrites.

ries or whether these categories represent extremes of a continuous variation of spines along more than one geometrical dimension. This question is easily addressed quantitatively, however. For example, the existence of sessile and thin spines as discrete types implies both a bimodality in the distribution of spine lengths and an inverse relationship between spine length and stalk diameter. Discrete categories are one of a number of possible schemes which might be useful in describing the variation among spines, however. A particularly simple and attractive alternative set of schemes are those involving geometrical invariance relationships. For example, membrane surface area of spines might always be constant (or at least much less variable than other pa- rameters such as stalk diameter or spine volume). Variations among spines then would represent alternate distributions of membrane between the head and stalk and constant surface area distortions of each compartment. This scheme is of special interest because it suggests that all spines would have about the same amount of plasma membrane, but other invariance relationships, such as constant volume or constant volume-to-surface area ratio, are also possible candidates for general schemes of spine geometry. All of these simple hypotheses, including discrete spine types, predict either multimodal distributions of spine shape parameters or correlations between shape parameters or both. In the absence of these, each independently varying parameter of spine shape must be 

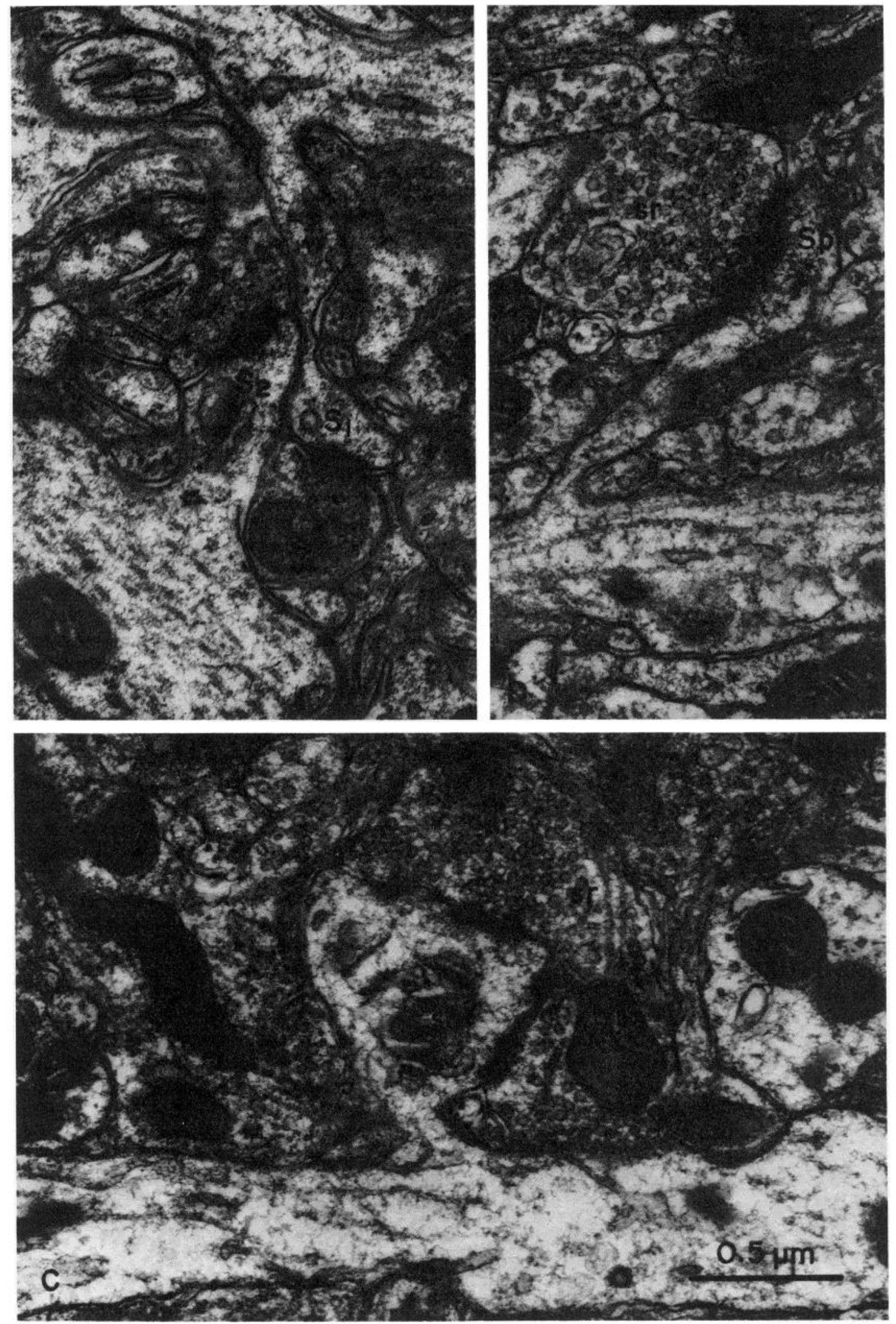

Figure 5. Variety of spine morphology observed in conventional thin sections from rat neostriatum. $a$, Short sessile $\left(S_{2}\right)$ and longer $\left(S_{l}\right)$ dendritic spines contacted by boutons containing small, round synaptic vesicles and forming asymmetrical synaptic junctions $(s r) . b$, A long, pedunculated dendritic spine $(S p)$ with a pronounced and flattened head and a larger synaptic apposition of the same type. $c$, A large mushroom-shaped dendritic spine with a pronounced spine apparatus $(s a)$. A large bouton $(s r)$ forms both synaptic and desmosome-like junctions with the spine. 
considered separately and all spines must be members of a multidimensional continuum of spine morphology.

Quantitative analysis of spine shape. Quantitative estimates of spine membrane area and volume were best obtained from analysis of serial thin sections. The surface area, perimeter, and length of synaptic apposition were measured from each section through 46 serially reconstructed dendritic spines. From such measurements and the average section thickness for the series, estimates of spine head volume and surface area, spine neck volume and surface area, and synaptic membrane surface area were obtained. Also measured were average dendritic diameters, average neck diameter, and neck length.

An example from a part of a series used for quantitative analysis is shown in Figure 6 . A dendrite $(D)$ shown in that figure gives rise to two spines $\left(S_{1}\right.$ and $\left.S_{2}\right)$. Each of these receives a synaptic contact onto the spine head. region (from boutons $b_{1}$ and $b_{3}$ ). The boundaries used to define the spine head and neck are shown. For tangentially or obliquely sectioned synaptic appositions, such as the ones shown in Figure 6, a trigonometric correction for inclination in the section was employed.

Total dendritic spine surface area varied from 0.61 to $3.14 \mu \mathrm{m}^{2}$ with a mean surface area of $1.46 \mu \mathrm{m}^{2}$ for the total sample. Spine volume ranged from 0.04 to $0.33 \mu \mathrm{m}^{3}$ (mean $=0.12 \mu \mathrm{m}^{3}$ ). There was a strong linear correlation between spine volume and surface area $(r=0.92, d f=$ $90, p<0.01)$. Because of the compound geometry of the spine, this relationship was more meaningfully examined by separate analysis of the volume-to-surface area relationship of the spine head and stalk.

For spine heads, the relationship between volume and membrane surface area was even more precise than that obtained for the entire spine. This relationship was nonlinear and is shown in Figure $7 A$. In that figure, individual spines are represented by points. 'The solid line represents the relationship between surface area and volume expected for spheres of various diameters ranging from 0 to $1 \mu \mathrm{m}$. The excellent fit of the spine head data to this curve for spheres confirms the impression gained by HVEM examination of thick sections and suggests that estimates of head diameter may be gained from measurements of head membrane surface area or volume using the simple mensuration formulae for spheres. When calculations of this type were performed, the diameter estimates were in close agreement with those gained by measurement of the diameter of the spine head in the single section of the series in which it was the largest. Spine head size, whether measured by volume, surface area, or maximal diameter, was distributed in a continuous fashion with no indication of bimodality or the existence of separate classes of spines based on head diameter.

Spine stalks, considered in isolation from the heads, also contributed to the correlation between volume and surface area of spines $(r=0.78, d f=90, p<0.01)$. Because both the volume and surface area of cylinders are dependent upon both their length and diameter, such variability would be expected even if the spine stalks were perfect cylinders of variable length and diameter. The extent to which spine stalks can be treated as cylinders was tested by comparison of the volume-tosurface area ratio (which for cylinders is dependent only upon cross-sectional diameter) and the measured diam- elers of spine stalks. This comparison is shown in Figure $7 B$. The slope of the linear relationship obtained differed considerably from that predicted for uniform cylinders (solid line). This deviation is in the direction expected for distortions which produce variation in diameter along the length of a cylinder, as of course does occur along spine necks (e.g., Figs. 3 and 4). These observations indicate that spine stalks cannot be treated as cylinders for purposes of measurement, and variables such as stalk diameter and volume must be measured independently rather than derived from each other. There was likewise no significant correlation between stalk length and diameter, measured either from stereo HVEM or serial thin section images (for the sample from serial sections shown in Fig. $7 E, r=0.21, d f=90, p>0.01$ ).

The above data indicate that no simplification of spine morphology based either on spine types as described above or on geometrical invariance schemes can be fit to the variation observed for neostriatal dendritic spines. The lack of an inverse relationship between stalk length and diameter indicates that all possible combinations of stalk diameters and lengths occur with likelihoods determined simply by the individual distributions of these spine shape parameters. Thus, long spine stalks do not tend to be thinner than short stalks. Furthermore, no geometrical parameter of the spines can be considered less variable than the others. Spine volume and surface area both increase with increases in head diameter, stalk diameter, and stalk length, and these increases are, on the average, as expected from simple expansion of the stalk and/or head compartments of the spine with no change in overall shape. Finally, there was no systematic covariance of spine head and stalk parameters. A scatter diagram for head and stalk membrane area is shown in Figure $7 D$ and is typical of the results obtained in the comparison of spine heads and stalks along any of the measure dimensions.

In the absence of any simple set of dendritic spinc types, quantitative comparison of spines located in various parts of the dendritic tree required comparison along each of the independent parameters of dendritic spine morphology obtained from serial thin sections. Distance from the soma was estimated using dendritic diameter. In no case was there any indication of a systematic variation of spine shape or size in relation to position in the dendritic field or to dendritic diameter. A representative example showing the comparison of dendritic diameter and spine length is shown in Figure $7 F$.

A striking positive correlation was obtained in comparisons of synaptic appositional area with various measures of spine head size. An example showing the linear relationship between synaptic membrane area (defined as the area of the junctional membrane specialization) and total head membrane is shown in Figure $7 C(r=$ $0.90, d f=90, p<0.01)$. For the entire sample of 46 spines, the synaptic membrane on the spine head accounted for approximately one-eighth of the total head membrane and this ratio was maintained despite an approximately 20 -fold variation in both variables. Positive correlations also were obtained with other measures of spine head size. The relationship between spine head size and synaptic membrane area can easily be seen in individual examples, such as those shown in Figures 5 and 6. The 

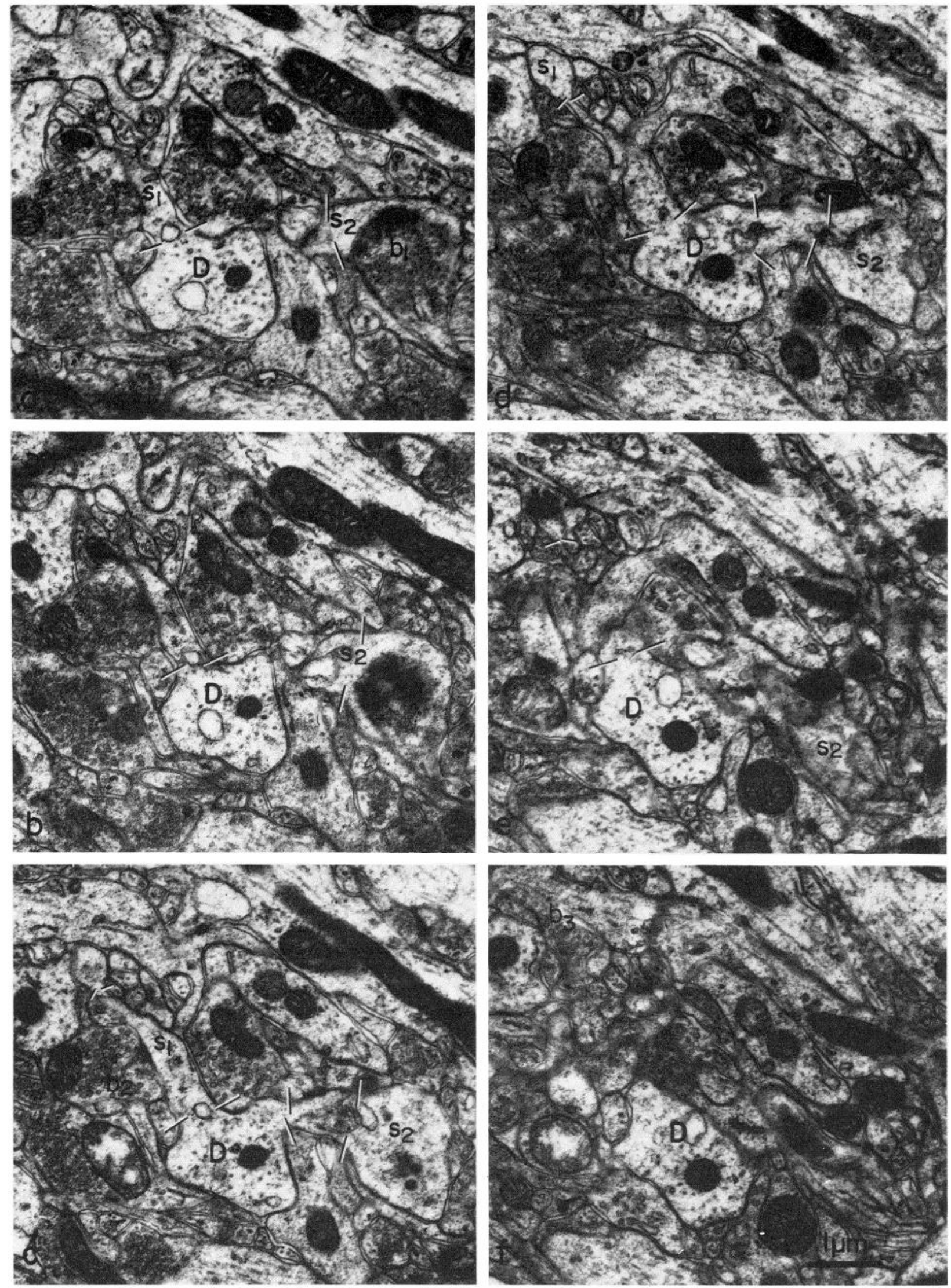

Figure 6. Selected sections from a series used for quantitative analysis of spine morphology. Boundaries used for head and stalk measurements are marked for two spines $\left(S_{1}\right.$ and $\left.S_{2}\right)$, arising from the same dendrite $(D)$. Both spines receive synaptic contacts from boutons with small round vesicles $\left(b_{1}\right.$ and $\left.b_{3}\right)$. One spine $\left(S_{l}\right)$ also receives a symmetrical contact from a bouton containing larger, more pleomorphic synaptic vesicles $\left(b_{2}\right)$. This contact is made onto the stalk of the spine. 

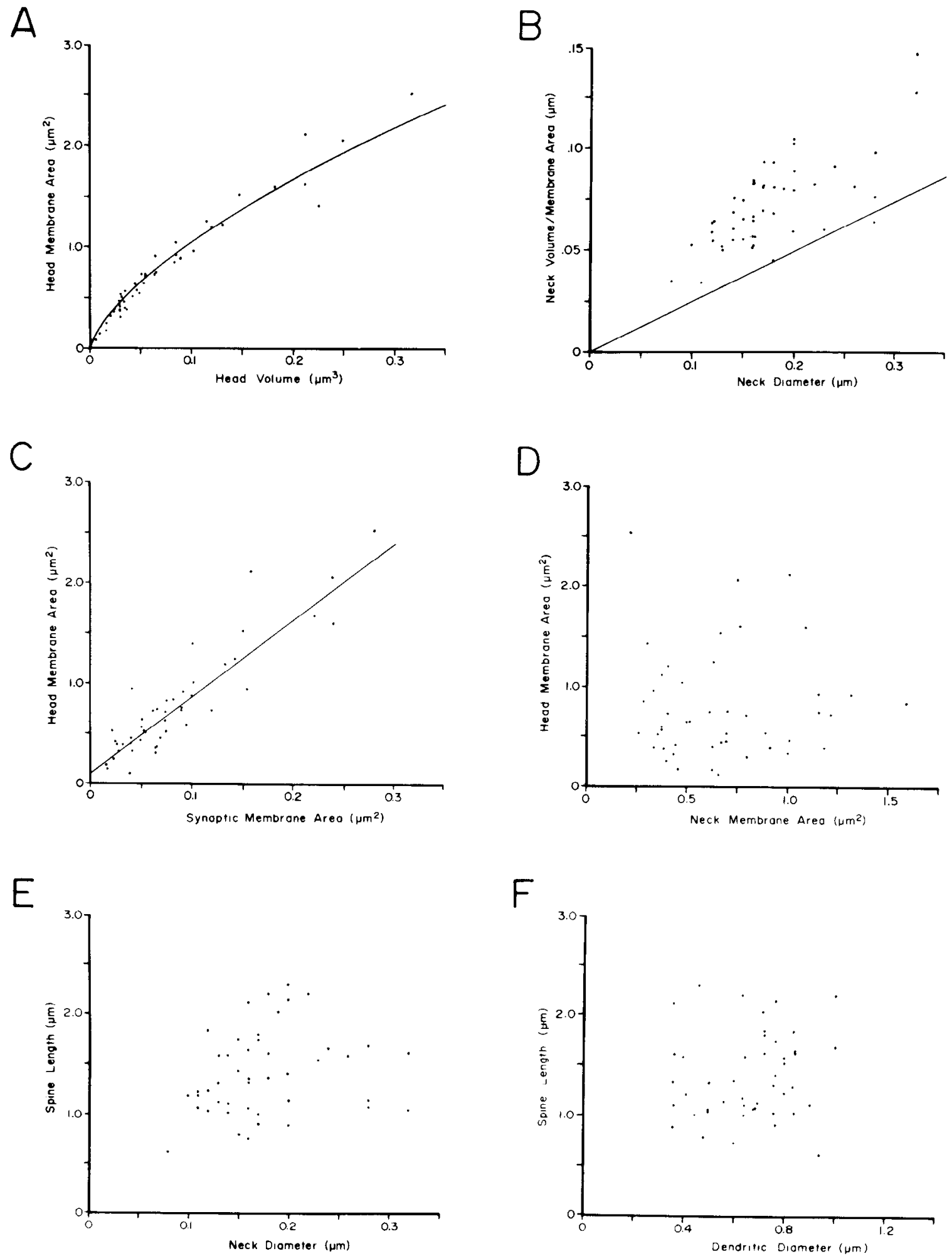

Figure 7. Relationships between spine shape parameters measured from serial sections through a sample of 46 dendritic spines examined in material such as that shown in Figure 6. $A$, A plot of membrane surface area versus enclosed volume for spine heads. The solid line is the locus of points expected for spherical bodies of various diameters. $B$, A plot of volume-to-surface area ratio versus neck diameter for spine stalks. The solid line shows the relationship expected for cylinders. The deviation of the spine stalks from this relationship can be seen. $C$, A plot comparing synaptic membrane surface area and surface area of the spine head membrane. The solid line is the best fitting regression line for these data. The slope of the line yields a ratio of $1 / 8$ for synaptic membrane versus total head membrane area. $D$ to $F$, Scatter plots showing the absence of any detectable relationship between head and stalk membrane surface areas, stalk length and diameter, and stalk length and dendritic diameter, respectively. 
dependence of synaptic membrane area on spine size was entirely restricted to the spine head, however. No relationship between synaptic membrane area and any spine stalk or dendritic parameter could be established (e.g., for the stalk membrane area and synaptic appositional area, $r=0.09, d f=90, p>0.01$ ).

Synaptic arrangements. A synaptic contact from a single bouton containing small round vesicles and forming an asymmetrical junctional specialization was observed on the head of every serially reconstructed dendritic spine in the present sample. This type of synapse has been shown to be characteristic of contacts made by extrinsic afferent fibers in neostriatum (Arluison et al., 1978; Groves, 1980; Hassler et al., 1978; Hattori et al., 1973; Kemp and Powell, 1971c). No spine received synaptic contacts from more than one bouton of this type, although a single bouton occasionally made contacts of irregular shape which might be interpreted in single sections as arising from more than one axon. On the largest spine heads, perforated synaptic contacts of the type described by Peters and Kaiserman-Abramof (1969) were sometimes observed. In 4 (8\%) of 46 spines examined in serial thin sections, a second synapse was present. In all cases, and in agreement with previous reports (Kemp and Powell, 1971a, b, d; Wilson and Groves, 1980), these contacts were not of the type formed by striatal afferents but contained pleomorphic synaptic vesicles and formed symmetrical junctional specializations. They made synapses on more proximal regions of the spine, either on the head near its junction with the stalk or onto the neck itself. An example is shown in Figure 6 (synapse made by $b_{2}$ ). In no case was a bouton of this type the only innervation of a dendritic spine, nor did any spine receive contacts from more than one element of each of these morphological types. There were no obvious differences in morphology between spines receiving only one synaptic contact and those receiving two.

Intracellular organization of the spine. Thin sections through dendritic spines in the rat neostriatum exhibited the characteristic cytoplasmic appearance of dendritic spines, containing only membranous saccules and tubules of spine apparatus and a seemingly random network of wispy filamentous material distributed throughout the cytoplasm. The spine apparatus, although present in all dendritic spines, varied greatly in degree of elaboration and position in the spine. In small, very thin spines, it was usually simple and tubular in form (e.g., Fig. $5 a$, spine $S_{1}$ ), while spines with large heads often exhibited large and elaborate laminar arrangements of saccules in the spine head and stalk (e.g., Fig. 5c). In serial sections, the spine apparatus usually could be traced to the tubular smooth endoplasmic reticulum of the dendrite. In the spine head, the spine apparatus often approached the postsynaptic membrane in the form of fine tubular profiles as described by Tarrant and Routtenberg (1979). In the spine stalk, the spine apparatus often appeared to nearly occlude the intracellular space. Because of the delicate, thin appearance of the spine apparatus membrane in comparison to the plasma membrane of the spine, portions of the complex not directly involved in the formation of parallel saccules were often difficult to identify in thin sections.
'The three-dimensional structure of the spine cytoplasm was most clearly observed in stereo electron micrographs of semithin sections such as those seen in Figures 8 to 10. In Figure 8, a spine head is shown with a cross-sectioned asymmetrical synaptic contact from a bouton with small, round synaptic vesicles. The postsynaptic membrane specialization is very pronounced and consists of a dense feltwork of filamentous and granular dense material which becomes gradually less tightly woven with distance from the postsynaptic membrane. Attached to this and radiating from it is a delicate lattice of filaments which corresponds to the wispy material seen in thin sections. These filaments range in thickness from about 3 to $10 \mathrm{~nm}$ and are attached both among themselves and to the surfaces of plasma and spine apparatus membranes (Figs. 8 to 10). Within the network can be seen at least two apparently different filamentous components. Some relatively straight and uniform filaments, 5 to $7 \mathrm{~nm}$ in diameter (e.g., Fig. 10), are identical to microfilaments observed elsewhere in the neurons and glia. Other strands are finer caliber and more variable, both in length and diameter. They are similar in appearance to cross-bridging structures observed in the cytoplasm of nearby axons and dendrites (e.g., Fig. 8). These two cytoplasmic components share a common organization in the spine. In the spine head, filaments have a primarily radial orientation, filling the head of the spine. The predominantly radial organization is shown most clearly in oblique sections through the synaptic specialization such as that shown in Figure 9. The synaptic contact in that figure is shown at an oblique angle. Spine head filaments attach to the membrane of the spine apparatus and to the plasma membrane of the spine over its entire length. Occasionally, filaments may be seen to course longitudinally into the spine stalk, but the predominant orientation is normal to the plasma membrane of the stalks. Thus, the networks of filaments present in the spine head and stalk are interconnected, but only at the junction of these two spine compartments, and not by a longitudinally oriented bundle of cytoskeletal elements running the entire length of the spine. Similarly, the cytoskeletal network of the spine stalk often is connected to filaments, tubules, and the membrane of the dendritic cytoplasm, but only near the junction of the spine and dendrite. In Figure 10 a junction of this type is seen in a semithin section. A dendritic microtubule is seen entering the dendritic spine and coursing partly into the spine stalk. Tubular elements of endoplasmic reticulum in the dendrite are continuous with the spine apparatus.

\section{Discussion}

A variety of indirect and theoretical considerations have led to general acceptance of the view that dendritic spines passively attenuate transmission at individual synapses by virtue of their high longitudinal resistance (Jack et al., 1975). In mammalian neostriatum, as in some other forebrain structures, the majority of excitatory afferent synapses are made onto dendritic spines of spiny projection neurons, while most axodendritic and axosomatic synapses subserve inhibitory intranuclear interactions (Frotscher et al., 1981; Kemp and Powell, 1971a, b, 

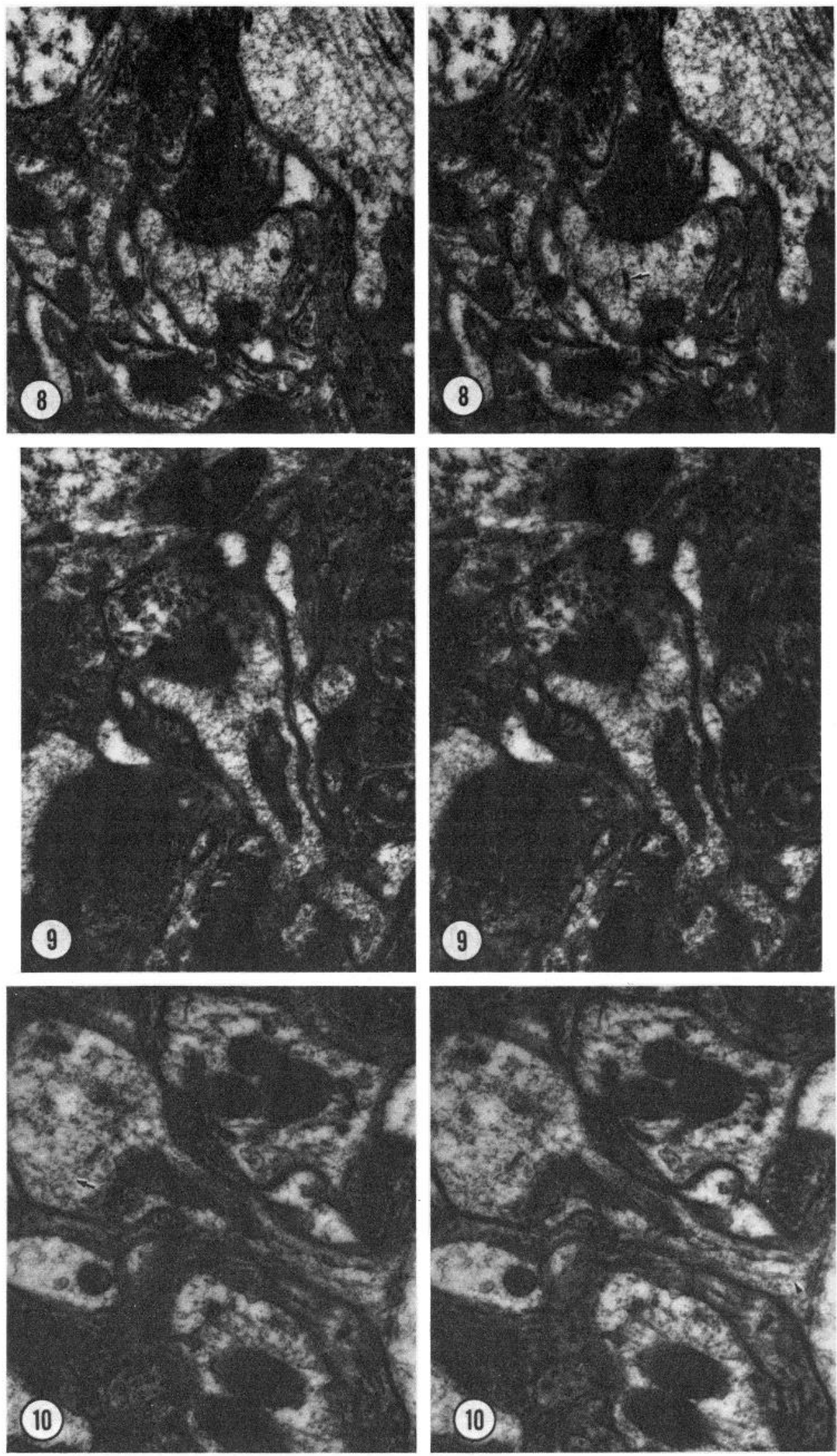

Figures 8 to 10. Stereo electron micrographs of semithin sections showing the organization of cytoskeletal elements within the dendritic spine cytoplasm. Figure 8. A spine head with synaptic contact is visible. The fine network of filaments associated with the postsynaptic web is apparent. A less tightly woven network of filaments is visible throughout the spine head cytoplasm, and these interconnect with postsynaptic membrane, spine plasma membrane, and spine apparatus. A portion of the spine apparatus is seen connected to both synaptic and nonsynaptic portions of the spine membrane by fine cytoplasmic filaments (arrow). 
c, d; Somogyi et al., 1981; Wilson and Groves, 1980). The distribution of dendritic spines on the dendrites, the shapes of the spines, and the relationship between spine geometry and synaptic location of presynaptic fiber origin thus may represent major influences on the integration of afferent activity in these structures. The overall sizes of spines and the spine shapes observed in the present study could partly be due to the procedures used in preparing them for visualization. A number of variations in those procedures have been examined and found to produce no measurable variation in the result. In all cases, however, anesthetized animals were perfused with chemical fixatives and the effects of these treatments on cell sizes and shapes have not been determined completely. Nonetheless, the range of spine sizes and shapes observed with these techniques suggests that spine geometry is highly variable. Furthermore, the observed variation is of a type which would be expected to produce major differences in the potency of otherwise identical synapses.

The wide range of spine head diameters observed in the present material may appear to be of questionable functional importance, since the high input impedance of the spine head in comparison to the stalk-dendritic combination gives changes in head diameter only a very small influence on the electrotonic properties of the spine (Rall, 1974). The finding that synaptic appositional area is very strongly correlated with spine head diameter suggests a functional relationship of a different lype. The area of synaptic apposition has been suggested to be directly related to the amount of transmitter released by a presynaptic action potential (Kuno et al., 1971, 1973). If such a relationship were to hold for the neostriatum, it would imply that the effectiveness of axospinous synapses could be predicted accurately from the diameter of the spine head. Such an influence of synaptic junctional size could be quite large, since an approximately 20 -fold variation in spine head and synaptic junctional area has been observed in the present sample.

Both the length and diameter of spine stalks are of critical importance in determining the input resistance of dendritic spines and, presumably, the current injected into the dendrite by a given synaptic conductance change. Both of these exhibited considerable variation in the present sample.

The simple distribution of dendritic spines on the dendrites of rat neostriatal neurons described here is in agreement with previous findings from cats (Kemp and Powell, 1971d) and monkeys (Pasik et al., 1976). To the extent that comparable published data from these species are available, they in fact suggest that the size and shape of the neurons, lengths of dendrites, and distribution of dendritic spines are indistinguishable across the three species. Apparent species differences in the overall density (but not distribution) of dendritic spines are small and as likely to be due to differences in spine counting technique employed by the different authors as to differences between species (Feldman and Peters, 1979). The shape of the spine density distribution along the dendrites of neostriatal neurons is in contrast to the complex distribution reported for apical dendrites of layer $\mathrm{V}$ pyramidal neurons in cerebral cortex (Marin-Padilla and Stibitz, 1968). That distribution along the pyramidal neuron has been proposed to reflect the superimposition of a number of simple distributions centered upon different layers and presumably representing inputs from different sources (Marin-Padilla and Stibitz, 1968; MarinPadilla et al., 1969). Because the major neostriatal afferents do not appear to terminate in a differential manner along the dendrites of the neostriatal spiny neuron, such complexities should perhaps not be expected in the distribution of spines on these neurons, and the distribution of dendritic spines should be identical in shape to the distribution of synaptic contacts from any of the major afferents.

Similarly, the absence of a clear spatial distribution of spines with different shapes would seem at variance with the situation described for other spiny neurons in the neocortex (Jones and Powell, 1969; Peters and Kaiserman-Abramof, 1970) and hippocampus (Laatch and Cowan, 1966). In these cases, dendritic spines have been reported to be smaller on large proximal dendrites and large on the most distal small dendrites. Rall (1974) has suggested that this relationship may act to maintain a constant ratio of dendritic spine to dendritic shaft input resistance and thus maintain equivalency of spine function in proximal and distal regions. This proposal relies on the importance of dendritic diameter in the relationship between spine shape and position in the dendritic tree. In neocortical and hippocampal spiny neurons, dendritic diameter, distance from the soma, cytoarchitectonic layer, and origin of afferent fibers are confounded. In neostriatum, the absence of any systematic variation of spine shape along the dendrites would seem to be inconsistent with any functionally necessary relationship between spine shape and dendritic diameter or distance from the soma. Instead it would appear most likely that the shape and size of dendritic spines is either random or determined by an interaction with individual afferent fibers making contacts on those spines. The observed variation in spine shapes on neostriatal neurons might thus represent differences between afferents of different origin (e.g., between spines receiving cortical versus thalamic fibers), or may represent variation among fibers of a common origin but differing history of activity, or both.

If the shape and size of dendritic spines are determined by some interaction with presynaptic fibers, more than one aspect of the presynaptic element must be expressed in the interaction, since different aspects of the dendritic spine vary independently of each other. The most con-

Figure 9. A nearly longitudinal section through a dendritic spine showing the radial organization of filaments in the dendritic spine cytoplasm. The synapse is shown in oblique section and the connection of the cytoskeleton to the postsynaptic web is visible. Figure 10. A longitudinal section through a dendritic spine. The synaptic contact is not included in this section, but a prominent spine apparatus is seen to advantage. The spine apparatus nearly fills the spine stalk near the junction with the spine head. Both very fine ( 4 to $5 \mathrm{~nm}$ ) filaments and larger microfilaments (arrow) can be seen in the spine head and stalk. The spine apparatus is continuous with the dendritic endoplasmic reticulum via a tubular element which runs parallel to a microtubule which enters the proximal portion of the spine from the dendrite (arrowhead). 
vincing case for such an interaction in the present material is presented by the strict relationship between the size of spine heads and the area of the synaptic junction. Since no relationship between any of the spine stalk parameters could be linked to the synaptic junctional area or to the size of the spine head, however, it is likely that spine stalks are shaped by influences at least partially independent of those determining the dimensions of the head. Uncoupled variation in spine head and stalk morphology recently has been reported to accompany long-term synaptic potentiation of transmission in the perforant pathway of the dentate gyrus (Fifková and Anderson, 1981).

The ultrastructural appearance of dendritic spines in the neostriatum and in other forebrain structures has been described by numerous authors using a variety of fixation techniques (Fifková and Van Harreveld, 1977; Gray, 1959; Jones and Powell, 1969; Kemp and Powell, 1971a; Mori, 1966; Pasik et al., 1976; Peters and Kaiserman-Abramof, 1969; 'Tarrant and Routtenberg, 1979; Westrum et al., 1980). In agreement with those studies, the cytoplasm of the neostriatal dendritic spine exhibits a remarkably stereotyped and specialized structure. Although in rare instances ribosomes, microtubules, and coated vesicles may be observed in dendritic spines, only three cytoplasmic structures are visible consistently: the spine apparatus, the postsynaptic junctional specialization, and a network of delicate wispy material of mostly undetermined composition.

The morphology of the spine apparatus is highly reminiscent of the smooth surfaced endoplasmic reticulum of the dendrite with which it can often be seen to be continuous. The elaboration into parallel flattened cisternae which occurs in many spines is a high surface area configuration which might, as suggested by Tarrant and Routtenberg (1979), represent a store of membrane available for changes in the surface area of the spine plasma membrane during dynamic changes in spine shape. No observations of intermediate stages in membrane exchange between spine apparatus and plasma membrane have been reported, however, and no evidence of exchange could be obtained in the present material. Furthermore, the most elaborate spine apparatuses generally are associated with spines possessing a large surface of plasma membrane, which does not suggest that the spine apparatus is depleted of membrane in large spines. The spine apparatus and its connection to the smooth endoplasmic reticulum also have been proposed as a route for the transfer of protein between spine and dendrite (Fifková and Van Harreveld, 1977). The spine apparatus also occupies a considerable proportion of the volume of the spine stalk and probably contributes substantially to the longitudinal resistance of the cytoplasm. Variation in spine apparatus position and volume might thus have an effect on the electrotonic properties of the spine. Finally, the similarity between the spine apparatus and the smooth endoplasmic reticulum suggests the possibility that it may act as a reservoir for calcium (Henkart et al., 1978). If dendritic spines do undergo dynamic changes in shape as a result of changes in synaptic input, such changes are most likely to involve calcium-dependent mechanisms similar to those generally held responsible for the control and maintenance of cell shape (Porter et al., 1979). The absence of mitochondria in dendritic spines in neostriatum is consistent with an exclusive role for the spine apparatus in the control of free calcium concentration in the cytoplasm of the spine. The spine apparatus, in conjunction with the other components of the dendritic spine cytoplasm, may thus function to control the size and shape of the spine.

The wispy filamentous component of the dendritic spine cytoplasm has been noted in most descriptions of spines based on examination of ultrathin sections. Occasionally, the presence of microfilaments also has been described, and in one report these were identified as actin on the basis of heavy meromyosin staining (Le Beaux and Willemot, 1975a, b). The combination of the calciumfrce stabilization buffer used in the present experiments and stereo electron microscopy of semithin sections has provided a three-dimensional view of the organization of this network in the spine cytoplasm. There are at least two cytoskeletal components participating in the formation of the delicate latticework filling the intracellular space of the spine. One of these is the microfilamentous network described by Le Beaux and Willemot (1975a, b). Microfilaments radiating from the postsynaptic density are seen throughout the spine head and connect to membranes in the spine. Although probably only a fraction of the total number of actin filaments present in the spine are visible in these preparations due to the destructive effects of osmium (Maupin-Szamier and Pollard, 1978), the radial organization of filaments observed in the present experiments is unlikely to be artifactual on this basis and thus probably represents an accurate image of the spatial organization of this cytoplasmic component. Microfilaments in the spine stalk likewise have a primarily radial rather than longitudinal orientation. Contraction along the directions of orientation of microfilaments in the dendritic spine thus would not be likely to produce a simple shortening of the spine or rounding up of its contours. Instead, an overall contraction of part or all of the spine would be more likely to occur, necessitating changes in both volume and surface area of the spine. Likewise, the orientation of microfilaments in the dendritic spine suggests no necessary mechanical linkage of head and stalk cytoskeleton. Also present in the dendritic spine cytoplasm and connecting both to membrane surfaces and to the microfilaments are finer filamentous structures of the type described as cross-linking microtubules and other organelles in neurons and other cells (Ellisman and Porter, 1980; Le Beaux and Willemot, 1975a, b; Metuzals et al., 1981; Porter et al., 1979; Wolosewick and Porter, 1978). In the absence of other organelles and in the constrained intracellular space of the dendritic spine, these elements connect microfilaments, spine apparatus, and plasma membrane to each other. Like the microfilaments, however, they appear more densely anchored in the postsynaptic density than other portions of the membrane. The preferential association of cytoskeletal components of the cytoplasm with postsynaptic membrane specialization has been reported previously (Cohen et al., 1980; Gulley and Reese, 1981; Le Beaux, 1973). In the dendritic spine, at least, this association may reflect a functional connection between cellular mechanisms involved in reception of synaptic signals and those responsible for cell shape. 


\section{References}

Arluison, M., Y. Agid, and F. Javoy (1978) Dopaminergic nerve endings in the neostriatum of the rat. I. Identification by intracerebral injections of 5-hydroxydopamine. Neuroscience $3: 657-673$.

Chang, H. -T. (1952) Cortical neurons with particular reference to the apical dendrites. Cold Spring Harbor Symp. Quant. Biol. 17: 189-201.

Chang, H. T., C. J. Wilson, and S. T. Kitai (1981) Single neostriatal efferent axons in the globus pallidus: A light and electron microscopic study. Science 213: 915-918.

Chung, J. W., R. Hassler, and A. Wagner (1977) Degeneration of two of nine types of synapses in the putamen after center median coagulation in the cat. Exp. Brain Res. 28: 345-361.

Cohen, R. S., J. J. Wolosewick, R. P. Becker, and G. D. Pappas (1980) The fine structure of synapses in specimens embedded in an extractable material (polyethylene glycol). J. Cell Biol. 87: 80a.

Coss, R. G., and A. Globus (1978) Spine stems on tectal interneurons in jewel fish are shortened by social stimulation. Science 200: 787-790.

Diamond, J., E. G. Gray, and G. M. Yasargil (1970) The function of the dendritic spine: An hypothesis. In Excitatory Synaptic Mechanisms, P. Andersen and J. K. S. Jansen, eds., pp. 213222, Universitet forlaget, Oslo.

DiFiglia, M., P. Pasik, and T. Pasik (1976) A Golgi study of neuronal types in the neostriatum of monkeys. Brain Res. 114: $245-256$.

Ellisman, M. H., and K. R. Porter (1980) Microtrabecular structure of the axoplasmic matrix: Visualization of crosslinking structures and their distribution. J. Cell Biol. 87: 464479.

Favard, P., and N. Carasso (1973) The preparation and observation of thick biological sections in the high voltage electron microscope. J. Microsc. 97: 59-81.

Feldman, M. L., and A. Peters (1979) A technique for estimating total spine numbers on Golgi-impregnated dendrites. J. Comp. Neurol. 118: 527-542.

Fifková, E., and C. L. Anderson (1981) Stimulation-induced changes in dimensions of stalks of dendritic spines in the dentate molecular layer. Exp. Neurol. 74: 621-627.

Fifková, E., and A. Van Harreveld (1977) Long-lasting morphological changes in dendritic spines of dentate granular cells following stimulation of the entorhinal area. J. Neurocytol. 6: $211-230$.

Fox, C. A., A. N. Andrade, D. E. Hillman, and R. C. Schwyn (1971/1972) The spiny neurons in the primate striatum: A Golgi and electron microscopic study. J. Hirnforsch. 13: 181201.

Frotscher, M., U. Rinne, R. Hassler, and W. Wagner (1981) Termination of cortical afferents on identified neurons in the caudate nucleus of the cat. Exp. Brain Res. 41: 329-337.

Globus, A., and A. B. Scheibel (1967) Synaptic loci in visual cortical neurons of the rabbit: The specific afferent radiation. Exp. Neurol. 18: 116-131.

Gray, F. G. (1959) Axo-somatic and axo-dendritic synapses of the cerebral cortex: An electron microscopic study. J. Anat. 93: 420-433.

Groves, P. M. (1980) Synaptic endings and their postsynaptic targets in neostriatum: Synaptic specializations revealed from analysis of serial sections. Proc. Natl. Acad. Sci. U. S. A. 77: 6926-6929.

Gulley, R. L., and T. S. Reese (1981) Cytoskeletal organization at the postsynaptic complex. J. Cell Biol. 91: 298-302.

Hassler, R., J. W. Chung, U. Rinne, and A. Wagner (1978) Selective degeneration of two out of nine types of synapses in cat caudate nucleus after cortical lesions. Exp. Brain Res. 31: $67-80$.
Hattori, T., H. C. Fibiger, P. L. McGeer, and L. Maler (1973) Analysis of the dopaminergic nigrostriatal projection by electron microscopic autoradiography. Exp. Neurol. 41: 599-611.

Henkart, M., T. S. Reese, and F. J. Brinley (1978) Endoplasmic reticulum sequesters $\mathrm{Ca}^{++}$in squid giant axon. Science 202:1300-1303.

Jack, J. J. B., D. Noble, and R. W. Tsien (1975) Electric Current Flow in Excitable Cells, pp. 218-223, Oxford University Press, London.

Jones, E. G., and T. P. S. Powell (1969) Morphological variations in the dendritic spines of the neocortex. J. Cell Sci. 5: 509-529.

Kemp, J. M., and T. P. S. Powell (1971a) The structure of the caudate nucleus of the cat: Light and electron microscopy. Philos. Trans. R. Soc. Lond. (Biol.) 262: 383-401.

Kemp, J. M., and T. P. S. Powell (1971b) The synaptic organization of the caudate nucleus. Philos. Trans. R. Soc. Lond. (Biol.) 262: 403-412.

Kemp, J. M., and T. P. S. Powell (1971c) The site of termination of afferent fibres in the caudate nucleus. Philos. Trans. R. Soc. Lond. (Biol.) 262: 413-427.

Kemp, J. M., and T. P. S. Powell (1971d) The termination of fibres from the cerebral cortex and thalamus upon dendritic spines in the caudate nucleus: A study with the Golgi method. Philos. Trans. R. Soc. Lond. (Biol.) 262: 429-439.

Kuno, M., S. A. Trukanis, and J. N. Weakly (1971) Correlation between nerve terminal size and transmitter release at the neuromuscular junction of the frog. J. Physiol. (Lond.) 213: $545-556$.

Kuno, M., E. J. Muñoz-Martinez, and M. Randić (1973) Synaptic action on Clarke's column neurones in relation to afferent terminal size. J. Physiol. (Lond.) 228: 343-360.

Laatch, R. H., and W. M. Cowan (1966) Electron microscopic studies of the dentate gyrus of the rat. I. Normal structure with special reference to synaptic organization. J. Comp. Neurol. 128: 359-396.

Langford, L. A., and R. E. Coggeshall (1979) Polassium ferricyanide: An improvement in neural fixation. Soc. Neurosci. Abstr. 5: 430.

Le Beaux, Y. J. (1973) An ultrastructural study of the synaptic densities, nematosomes, neurotubules, neurofilaments and of a further three-dimensional filamentous network as disclosed by the E-PTA staining procedure. Z. Zellforsch. Mikrosk. Anat. 143: 239-272.

Le Beaux, Y. J., and J. Willemot (1975a) An ultrastructural study of the microfilaments in rat brain by means of heavy meromyosin labeling. I. The Perikaryon, the dendrites and the axon. Cell Tissue Res. 160: 1-36.

Le Beaux, Y. J., and J. Willemot (1975b) An ultrastructural study of the microfilaments in rat brain by means of heavy meromyosin labeling. II. The synapses. Cell Tissue Res. 160: $37-68$.

Lee, K. S., F. Schottler, M. Oliver, and G. Lynch (1980) Brief bursts of high frequency stimulation produce two types of structural changes in rat hippocampus. J. Neurophysiol. 44: 247-258.

Llinás, R. R., and D. E. Hillman (1969) Physiological and morphological organization of the cerebellar circuits in various vertebrates. In Neurobiology of Cerebellar Evolution and Development, R. Llinás, ed., pp. 43-73, AMA-ERF Institute for Biomedical Research, Chicago.

Marin-Padilla, M., and G. R. Stibitz (1968) Distribution of the apical dendritic spines of the layer $V$ pyramidal cells of the hamster neocortex. Brain Res. 11: 580-592.

Marin-Padilla, M., G. R. Stibitz, C. P. Almy, and H. N. Brown (1969) Spine distribution of the layer $V$ pyramidal cells in man: A cortical model. Brain Res. 12: 493-496.

Maupin-Szamier, P., and T. D. Pollard (1978) Actin filament destruction by osmium tetroxide. J. Cell Biol. 72: 837-852. 
Metuzals, J., V. Montpetit, and D. F. Clapin (1981) Organization of the neurofilamentous network. Cell Tissue Res. 214: 455482.

Mori, S. (1966) Some observations on the fine structure of the corpus striatum of rat brain. Z. Zellforsch. Mikrosk. Anat. 70: 461-488.

Pasik, P., T. Pasik, and M. DiFiglia (1976) Quantitative aspects of neuronal organization in the neostriatum of the macaque monkey. In The Basal Ganglia, M. D. Yahr, ed., pp. 57-88, Raven Press, New York.

Peters, A., and I. R. Kaiserman-Abramof (1969) The small pyramidal neuron of the rat cerebral cortex. The synapses upon dendritic spines. Z. Zellforsch. Mikrosk. Anat. 100: 487506.

Peters, A., and I. R. Kaiserman-Abramof (1970) The small pyramidal neuron of the rat cerebral cortex. The perikaryon, dendrites and spines. Am. J. Anat. 127: 321-356.

Porter, K. R., H. R. Byers, and M. H. Ellisman (1979) The cytoskeleton. In The Neurosciences: Fourth Study Program, F. O. Schmitt and F. G. Worden, eds., pp. 703-722, MIT Press, Cambridge, MA.

Preston, R. J., G. A. Bishop, and S. T. Kitai (1980) Medium spiny projection from rat striatum: An intracellular horseradish peroxidase study. Brain Res. 183: 253-263.

Purpura, D. P. (1974) Dendritic spine "dysgenesis" and mental retardation. Science 186: 1126-1128.

Rall, W. (1974) Dendritic spines, synaptic potency and neuronal plasticity. In Cellular Mechanisms Subserving Changes in Neuronal Activity, C. D. Woody, K. A. Brown, T. J. Crow, and J. D. Knispel, eds., pp. 13-21, Brain Information Service, UCLA, Los Angeles.

Ramon y Cajal, S. (1911) Histologie du Systeme Nerveux de l'Homme et des Vertebres, Vol. 1, pp. 66-70, Maloine, Paris.

Shepherd, G. M., and R. K. Brayton (1979) Computer simulation of a dendrodendritic synaptic circuit for self- and lateralinhibition in the olfactory bulb. Brain Res. 175: 377-382.

Somogyi, P., J. P. Bolam, and A. D. Smith (1981) Monosynaptic cortical input and local axon collaterals of identified striatonigral neurons. A light and electron microscopic study using the Golgi-peroxidase transport-degeneration procedure. J. Comp. Neurol. 195: 567-584.

Tarrant, S. B., and A. Routtenberg (1977) The synaptic spinule in the dendritic spine: Electron microscopic study of the hippocampal dentate gyrus. Tissue Cell 9: 461-473.

Tarrant, S. B., and A. Routtenberg (1979) Postsynaptic membrane and spine apparatus: Proximity in dendritic spines. Neurosci. Lett. 11: 289-294.

Valverde, F. (1967) Apical dendritic spines of the visual cortex and light deprivation in the mouse. Exp. Brain Res. 3: 337352.

Van Harreveld, A., and E. Fifková (1975) Swelling of dendritic spines in fascia dentata after stimulation of the perforant fibers as a mechanism of posttetanic potentiation. Exp. Neurol. 49: 736-749.

Venable, J. H., and R. Coggeshall (1965) A simplified lead citrate stain for use in electron microscopy. J. Cell Biol. 25: 407.

Westrum, L. E., D. H. Jones, E. G. Gray, and J. Barron (1980) Microtubules, dendritic spines and spine apparatuses. Cell Tissue Res. 208: 171-181.

Wilson, C. J., and P. M. Groves (1979) A simple and rapid section embedding technique for sequential light and electron microscopic examination of individually stained central neurons. J. Neurosci. Methods 1: 383-391.

Wilson, C. J., and P. M. Groves (1980) Fine structure and symaptic connections of the common spiny neuron of the rat neostriatum: A study employing intracellular injection of horseradish peroxidase. J. Comp. Neurol. 194: 599-616.

Wilson, C. J., and P. M. Groves (1981) Spontaneous firing patterns of identified spiny neurons in the rat neostriatum. Brain Res. 220: 67-80.

Wilson, C. J., H. T. Chang, and S. T. Kitai (1982) Origins of postsynaptic potentials evoked in identified rat neostriatal neurons by stimulation in substantia nigra. Exp. Brain Res., 45: $157-167$.

Wolosewick, J. J., and K. R. Porter (1978) The microtrabecular lattice of the cytoplasmic ground substance: Artifact or reality. J. Cell Biol. 82:114-139. 